\title{
Nanoscale
}

A) Check for updates

Cite this: Nanoscale, 2018, 10, 6212

DOI: $10.1039 / c 8 n r 90055 h$

rsc.li/nanoscale

\section{Correction: A smart pH-responsive nano-carrier as a drug delivery system for the targeted delivery of ursolic acid: suppresses cancer growth and metastasis by modulating P53/MMP-9/PTEN/ CD44 mediated multiple signaling pathways}

Kai Jiang, Ting Chi, Tao Li, Guirong Zheng, Lulu Fan, Yajun Liu, Xiufen Chen, Sijia Chen, Lee Jia and Jing-Wei Shao (D) *

Correction for 'A smart $\mathrm{pH}$-responsive nano-carrier as a drug delivery system for the targeted delivery of ursolic acid: suppresses cancer growth and metastasis by modulating P53/MMP-9/PTEN/CD44 mediated multiple signaling pathways' by Kai Jiang et al., Nanoscale, 2017, 9, 9428-9439.

The authors wish to correct Fig. 5(A), 7 and 8(C) as they contained incorrect images and data in the original published version of their manuscript. The correct versions are provided below. The authors confirm that the changes do not affect the scientific conclusions of the published work.

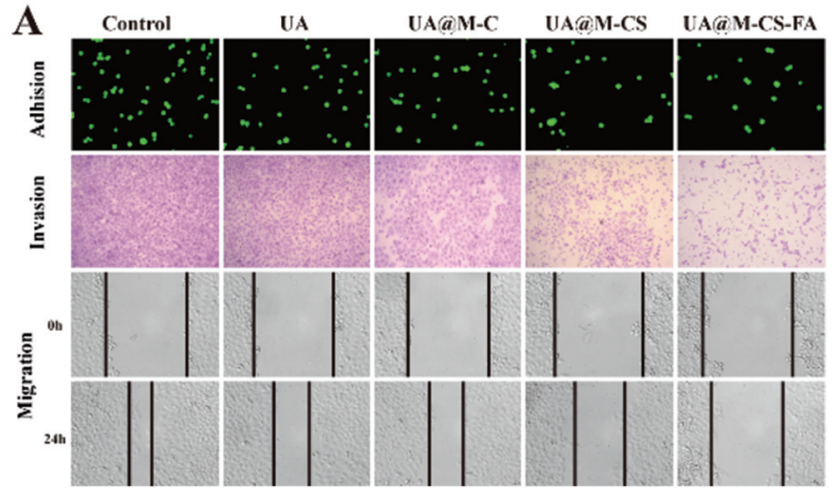

Fig. 5 (A): HeLa cell representative images of adhesion, invasion, and scratch assays. 

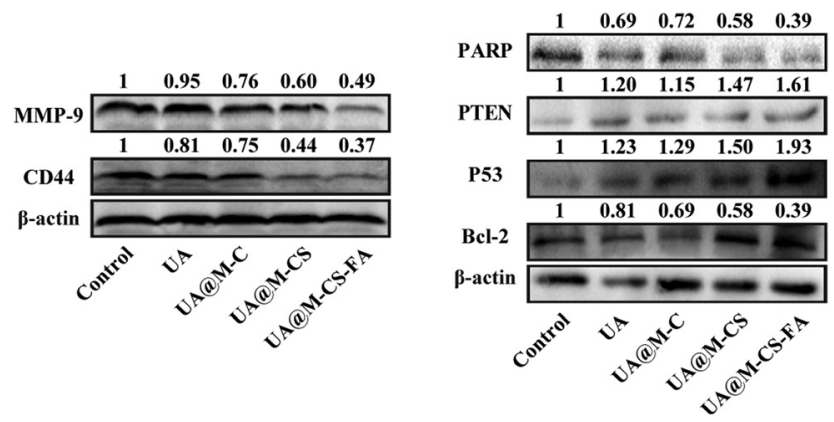

Fig. 7 Western blot analysis of PARP, PTEN, P53, Bcl-2, MMP-9 and CD44 expressions after HeLa cells were treated with free UA, UA loaded nanoparticles for $24 \mathrm{~h}$.

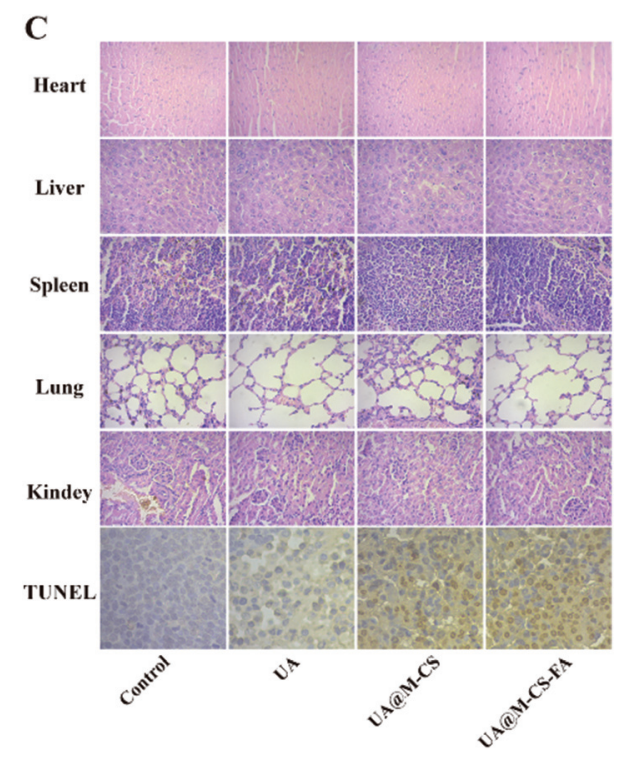

Fig. 8 (C): Histopathological analysis of the heart, liver, spleen, lung and kidney stained with hematoxylin and eosin, and tumors with TUNEL staining after treatment with PBS, UA, UA@M-CS, and UA@M-CS-FA.

The Royal Society of Chemistry apologises for these errors and any consequent inconvenience to authors and readers. 